VOL. 38 (1988) [125-130]

\title{
UNIMODAL EXPANDING MAPS OF THE INTERVAL
}

\author{
BAU-SEN DU
}

\begin{abstract}
Let $I=[0,1]$ and let $f$ be an unimodal expanding map in $C^{0}(I, I)$. If $f$ has an expanding constant $\lambda \geqq\left(\lambda_{n}\right)^{1 / 2^{m}}$ for some integers $m \geqq 0$ and $n \geqq 1$, where $\lambda_{n}$ is the unique positive zero of the polynomial $x^{2 n+1}-2 x^{2 n-1}-1$, then we show that $f$ has a periodic point of period $2^{m}(2 n+1)$. The converse of the above result is trivially false. The condition $\lambda \geqq\left(\lambda_{n}\right)^{1 / 2^{m}}$ in the above result is the best possible in the sense that we cannot have the same conclusion if the number $\lambda_{n}$ is replaced by any smaller positive number and the generalisation of the above result to arbitrary piecewise monotonic expanding maps in $C^{0}(I, I)$ is not possible.
\end{abstract}

\section{INTRODUCTION}

Let $I$ denote the unit interval $[0,1]$ of the real line and let $f \in C^{0}(I, I)$. For any positive integer $n$, let $f^{n}$ denote the $n$th iterate of $f$. A point $x_{0} \in I$ is called a periodic point of $f$ if $f^{m}\left(x_{0}\right)=x_{0}$ for some positive integer $m$ and the smallest such positive integer $m$ is called the period of $x_{0}$ (under $f$ ).

The continuous map $f$ is said to be piecewise monotonic if $I$ can be divided into finite number of non-degenerate subintervals $I_{1}, I_{2}, \ldots, I_{k}$ on each of which $f$ is either strictly increasing or strictly decreasing. If $f$ is piecewise monotonic and there is a constant $\lambda>1$ such that $|f(x)-f(y)| \geqq \lambda|x-y|$ whenever both $x$ and $y$ belong to some interval on which $f$ is monotonic, then we call $f$ an expanding map and, in this case, call $\lambda$ an expanding constant for $f$.

The main result of this note is the following

Theorem. Assume that $f \in C^{0}(I, I)$ is an unimodal expanding map. If $f$ has an expanding constant $\lambda \geqq\left(\lambda_{n}\right)^{1 / 2^{m}}$ for some integers $m \geqq 0$ and $n \geqq 1$, where $\lambda_{n}$ is the unique positive zero of the polynomial $x^{2 n+1}-2 x^{2 n-1}-1$, then $f$ has a periodic point of period $2^{m}(2 n+1)$.

The above result improves the main result in [4] and answers the question posed in $[6$, p. 437].

Received 22 October 1987

Copyright Clearance Centre, Inc. Serial-fee code: 0004-9729/88 \$A2.00+0.00. 
Remark 1. It is easy to see that the converse of the above result is false. In the following, we present one such example. For $1 / 2<\alpha \leqq 1$, let $g_{\alpha}$ be the map in $C^{0}(I, I)$ defined by letting $g_{\alpha}(x)=2 x$ for $0 \leqq x \leqq 1 / 2$ and $g_{\alpha}(x)=-2 \alpha x+\alpha+1$ for $1 / 2 \leqq x \leqq 1$. Then it is clear that $g_{\alpha}$ is an unimodal expanding map with $2 \alpha$ as an expanding constant which is close to 1 when $\alpha$ is close to $1 / 2$. However, it is shown in [7] that, for $1 / 2<\alpha \leqq 1, g_{\alpha}$ has a periodic point of period 6 .

Remark 2. The above result is the best possible in the sense that, if $m \geqq 0$ and $n \geqq 1$ are integers and $\lambda$ is a real number with $1<\lambda<\left(\lambda_{n}\right)^{1 / 2^{m}}$, where $\lambda_{n}$ is the unique positive zero of the polynomial $x^{2 n+1}-2 x^{2 n-1}-1$, then there is an unimodal expanding map in $C^{0}(I, I)$ with $\lambda$ as an expanding constant which has no periodic point of period $2^{m}(2 n+1)$. To give one such example, let, for $1<\lambda \leqq 2, f_{\lambda}$ be the unimodal expanding map in $C^{0}(I, I)$ defined by letting $f_{\lambda}(x)=\lambda x+2-\lambda$ for $0 \leqq x \leqq 1-1 / \lambda$ and $f_{\lambda}(x)=-\lambda x+\lambda$ for $1-1 / \lambda \leqq x \leqq 1$. Then it is shown in [10, p. 227] that $f_{\lambda}$ has a periodic point of period $2^{m}(2 n+1)$ where $m \geqq 0$ and $n \geqq 1$ are integers if and only if $\lambda \geqq\left(\lambda_{n}\right)^{1 / 2^{m}}$

Remark 3. The above result does not hold for arbitrary piecewise monotonic expanding maps in $C^{0}(I, I)$. 'To be more precise, there exist, for integers $m \geqq 1$ and $n \geqq 2$, piecewise monotonic expanding maps $f_{m, n}$ in $C^{0}(I, I)$ such that (i) the integer $n$ is an expanding constant for $f_{m, n}$; (ii) the topological entropy (see [1] for definition) of $f_{m, n}$ is greater than or equal to $\log n$; and (iii) $f_{m, n}$ has periodic points of period $2^{m} \cdot 3$, but no periodic points of period $2^{m-1}(2 k+1)$ for any positive integer $k$. See [8] for some examples.

\section{Preliminary Results}

We now introduce some preliminary results which will be used in the proof of our main result. The following result is a well-known result of Sharkovskii ([14], see also $[\mathbf{2}, \mathbf{3}, \mathbf{5}, \mathbf{9}, \mathbf{1 3}, \mathbf{1 5}, \mathbf{1 6}])$.

THEOREM 1. Rearrange the set of all positive integers according as the following new ordering (called Sharkovskii's ordering): $3 \rightarrow 5 \rightarrow 7 \rightarrow \ldots \rightarrow 2 \cdot 3 \rightarrow 2 \cdot 5 \rightarrow$ $2 \cdot 7 \rightarrow \ldots \rightarrow 2^{k} \cdot 3 \rightarrow 2^{k} \cdot 5 \rightarrow 2^{k} \cdot 7 \rightarrow \ldots \rightarrow 2^{3} \rightarrow 2^{2} \rightarrow 2 \rightarrow 1$. Assume that $f \in C^{0}(I, I)$ has a periodic point of period $m$. Then $f$ also has a periodic point of period $n$ precisely when $m \rightarrow n$

The following result $([11,12]$ of $\mathrm{Li}$ et al is useful in showing the existence of periodic points of certain odd periods $>1$.

LEMMA 2. Let $f \in C^{0}(I, I)$ and let $n \geqq 3$ be an odd integer. If there is a point $x_{0}$ such that $f^{n}\left(x_{0}\right) \leqq x_{0}<f\left(x_{0}\right)$ or $f^{n}\left(x_{0}\right) \geqq x_{0}>f\left(x_{0}\right)$, then $f$ has a periodic point of period $n$. 
A proof of the following result can be found in [17].

Lemma 3. Assume that $f \in C^{0}(I, I)$ is a piecewise monotonic expanding map with $\lambda$ as an expanding constant. Then, for every positive integer $m, f^{m}$ is a piecewise monotonic expanding map with $\lambda^{m}$ as an expanding constant.

The following result improves [4, Lemma 3].

LeMmA 4. Let $h \in C^{0}(I, I)$ be a piecewise monotonic expanding map with $\lambda$ as an expanding constant. Assume that $h$ admits a point $y$ with $h(y)<y<h^{2}(y)$ such that $h$ is decreasing on $[h(y), y]$ and increasing on $\left[y, h^{2}(y)\right]$. If $h$ has no periodic point of period 3 and $g=h^{2}$, then there is a point $z$ with $h(y)=g(z)<z<g^{2}(z)<y$ such that $g$ is decreasing on $[g(z), z]$ and increasing on $\left[z, g^{2}(z)\right]$.

ProOF: Since $h$ is strictly decreasing on $[h(y), y]$ and $y \in\left[h(y), h^{2}(y)\right]=$ $h([h(y), y])$, there is a unique point $z \in(h(y), y)$ such that $h(z)=y$. So, $g(z)=$ $h(y)<z$. If $g^{2}(z) \geqq y$, then $h(y)<z<y \leqq g^{2}(z)=h^{3}(y)$. By Lemma 2 , $h$ has a periodic point of period 3 which is a contradiction. So, $h^{3}(y)=g^{2}(z)<y$. Since $g^{2}(z)-g(z)=h^{4}(z)-h^{2}(z) \geqq \lambda\left(h^{3}(z)-h(z)\right) \geqq \lambda^{2}\left(z-h^{2}(z)\right)>z-h^{2}(z)=z-g(z)$, we obtain $g^{2}(z)>z$. Consequently, we have shown that $h(y)=g(z)<z<g^{2}(z)<y$.

On the other hand, since $g_{\| g(z), z]}$ is the composition of $h_{\mid[g(z), z]}$ which is decreasing and $h_{\mid h([g(z), z])}$ which is increasing, it is decreasing. Similarly, since $g_{\mid\left[z, g^{2}(z)\right]}$ is the composition of $h_{\left.\| z, g^{2}(x)\right]}$ which is decreasing and $h_{\mid h\left(\left|z, g^{2}(z)\right|\right)}$ which is also decreasing, it is increasing. This completes the proof.

The following lemma is crucial in proving our main result.

Lemma 5. Assume that $f \in C^{0}(I, I)$ admits a point $y$ with $f(y)<y<f^{2}(y)$ such that $f$ is decreasing on $[f(y), y]$ and increasing on $\left[y, f^{2}(y)\right]$. For every positive integer $k$, let $\lambda_{k}$ denote the unique positive zero of the polynomial $x^{2 k+1}-2 x^{2 k-1}-1$. If, for some positive integer $n,|f(u)-f(v)| \geqq \lambda_{n}|u-v|$ whenever both $u$ and $v$ lie in $[f(y), y]$ or in $\left[y, f^{2}(y)\right]$, then $f$ has a periodic point of period $2 n+1$.

PROOF: If $f^{3}(y) \geqq f^{2}(y)$, then it is clear that $f$ has a periodic point of period 3 and hence, by Theorem $1, f$ has a periodic point of period $2 n+1$. So, we assume that $f^{3}(y)<f^{2}(y)$. Note that, since $f$ is increasing on $\left[y, f^{2}(y)\right], f(y) \leqq f^{3}(y)$. For simplicity, we let $\lambda=\lambda_{n}$ in the sequel.

Assume that $n=1$. Then we have $f^{3}(y)-f(y) \geqq \lambda\left[f^{2}(y)-y\right]=$ $\lambda\left\{\left[f^{2}(y)-f(y)\right]-[y-f(y)]\right\} \geqq \lambda\{\lambda[y-f(y)]-[y-f(y)]\}=\lambda(\lambda-1)[y-f(y)]=$ $\left\{\left[\left(\lambda^{3}-2 \lambda-1\right) /(\lambda+1)\right]+1\right\}[y-f(y)]=y-f(y)$. So, $f^{3}(y) \geqq y$. By Lemma $2, f$ has a periodic point of period 3 .

Assume that $n \geqq 2$. If $f^{3}(y) \geqq y$, then by Lemma $2, f$ has a periodic point of period 3 , and hence, by Theorem $1, f$ has a periodic point of period $2 n+1$. So, we 
assume that $f^{3}(y)<y$. Consequently, $f$ maps $\left[f(y), f^{2}(y)\right]$ onto itself. On the other hand, $y-f^{4}(y)=\left[f^{2}(y)-f^{4}(y)\right]-\left[f^{2}(y)-y\right] \geqq\left(\lambda^{2}-1\right)\left[f^{2}(y)-y\right]>0$. Now, we have two cases to consider:

Case 1. $n=2$.

In this case, we have $f^{5}(y)-f(y) \geqq \lambda\left[y-f^{4}(y)\right] \geqq \lambda\left(\lambda^{2}-1\right)\left[f^{2}(y)-y\right]=$ $\lambda\left(\lambda^{2}-1\right)\left\{\left[f^{2}(y)-f(y)\right]-[y-f(y)]\right\} \geqq \lambda\left(\lambda^{2}-1\right)(\lambda-1)[y-f(y)]=$ $\left\{\left[\left(\lambda^{5}-2 \lambda^{3}-1\right) /(\lambda+1)\right]+1\right\}[y-f(y)]=y-f(y)$. So, $f^{5}(y) \geqq y$. By Lemma 2 , $f$ has a periodic point of period 5 .

Case 2. $n>2$.

In this case, if $f^{2 k+1}(y) \geqq y$ for some $1 \leqq k \leqq n-1$, then by Lemma 2 , $f$ has a periodic point of period $2 k+1$, and hence, by Theorem $1, f$ has a periodic point of period $2 n+1$. So we assume that $f^{2 k+1}(y)<y$ for all $1 \leqq k \leqq n-1$. Also, for all $2 \leqq k \leqq n$, we define $A_{k}(\lambda)$ recursively, by putting $A_{2}(\lambda)=\lambda^{2}-1$ and $A_{j+1}(\lambda)=\lambda^{2} A_{j}(\lambda)-1$ for $2 \leqq j \leqq n-1$. Then, since $\lambda=\lambda_{n} \geqq \sqrt{2}$, we have $A_{k}(\lambda) \geqq 1$ for all $2 \leqq k \leqq n$.

Assume that $y-f^{2 k}(y) \geqq A_{k}(\lambda)\left[f^{2}(y)-y\right]$ for some $2 \leqq k \leqq n-1$. Then

$$
\begin{aligned}
y-f^{2 k+2}(y) & =\left[f^{2}(y)-f^{2 k+2}(y)\right]-\left[f^{2}(y)-y\right] \\
& \geqq \lambda^{2}\left[y-f^{2 k}(y)\right]-\left[f^{2}(y)-y\right] \geqq \lambda^{2} A_{k}(\lambda)\left[f^{2}(y)-y\right]-\left[f^{2}(y)-y\right] \\
& =A_{k+1}(\lambda)\left[f^{2}(y)-y\right]>0
\end{aligned}
$$

Since we have already shown that $y-f^{4}(y) \geqq A_{2}(\lambda)\left[f^{2}(y)-y\right]$, the above implies, by induction on $k$, that $y>f^{2 n}(y)$. Consequently

$$
\begin{aligned}
f^{2 n+1}(y)-f(y) & \geqq \lambda\left[y-f^{2 n}(y)\right] \geqq \lambda A_{n}(\lambda)\left[f^{2}(y)-y\right] \\
& \geqq \lambda A_{n}(\lambda)\left\{\left[f^{2}(y)-f(y)\right]-[y-f(y)]\right\} \\
& \geqq \lambda A_{n}(\lambda)\{\lambda[y-f(y)]-[y-f(y)]\} \\
& =\lambda(\lambda-1) A_{n}(\lambda)[y-f(y)] \\
& =\left\{\left[\left(\lambda^{2 n+1}-2 \lambda^{2 n-1}-1\right) /(\lambda+1)\right]+1\right\}[y-f(y)] \\
& =y-f(y)
\end{aligned}
$$

Thus, $f^{2 n+1}(y) \geqq y$. By Lemma $2, f$ has a periodic point of period $2 n+1$.

This completes the proof.

\section{PRoOF OF THE THEOREM}

Without loss of generality, we may assume that there is a point $c$ with $0<c<1$ such that $f$ is increasing on $[0, c]$ and decreasing on $[c, 1]$. 
If $m=0$, the desired result follows from Lemma 5 above. So, from now on, we assume that $m \geqq 1$. Since $f(c)-f^{2}(c) \geqq \lambda[f(c)-c]>f(c)-c$, we have $f^{2}(c)<c$. If $f^{3}(c) \leqq c$, then since $f^{3}(c) \leqq c<f(c), f$ has, by Lemma 2, a periodic point of period 3. By Theorem 1, $f$ has a periodic point of period $2^{m}(2 n+1)$. So, we assume that $f^{3}(c)>c$. Since $f^{4}(c)-f^{2}(c) \geqq \lambda^{2}\left(c-f^{2}(c)\right)>c-f^{2}(c)$, if follows that $f^{2}(c)<c<f^{4}(c)$.

If $f^{5}(c) \leqq c$, then since $f^{5}(c) \leqq c<f(c), f$ has, by Lemma 2, a periodic point of period 5 . By Theorem $1, f$ has a periodic point of period $2^{m}(2 n+1)$. So, we assume that $f^{5}(c)>c$. Since $\left.f^{2}\right|_{\left[f^{2}(c), c\right]}$ is the composition of $\left.f\right|_{\left[f^{2}(c), c\right]}$ which is increasing and $\left.f\right|_{f\left(\left[f^{2}(c), c\right]\right)}$ which is decreasing, it is decreasing. Similarly, since $\left.f^{2}\right|_{\left[c, f^{4}(c)\right]}$ is the composition of $\left.f\right|_{\left[c, f^{4}(c)\right]}$ which is decreasing and $\left.f\right|_{f\left(\left[c, f^{4}(c)\right]\right)}$ which is also decreasing, it is increasing. If $f^{2}$ has a periodic point of period 3 , then, by Theorem $1, f$ has a periodic point of period $2^{m}(2 n+1)$. Otherwise, we can apply Lemma 4 to the map $h=f^{2}$ with $y=c$. So, without loss of generality, we may assume that $f$ has no periodic point of period $2^{k} \cdot 3$ for any integer $k$ with $0<k<m$ and let $g=f^{2^{m}}$, By Lemma 4, there is a point $z$ with $f^{2}(c)=g(z)<z<g^{2}(z)<c$ such that $g$ is decreasing on $[g(z), z]$ and increasing on $\left[z, g^{2}(z)\right]$. By Lemma $3, g$ is a piecewise monotonic expanding map in $C^{0}(I, I)$ with $\lambda^{2^{m}}$ as an expanding constant. Since $\lambda^{2^{m}} \geqq \lambda_{n}$, it follows from Lemma 5 that $g$ has a periodic point of period $2 n+1$. Therefore, by Theorem $1, f$ has a periodic point of period $2^{m}(2 n+1)$. This completes the proof.

\section{REFERENCES}

[1] R. Adler, A. Konheim and M. McAndrew, 'Topological entropy', Trans. Amer. Math. Soc. 114 (1965), 309-319.

[2] L. Block, J. Guckenheimer, M. Misiurewicz and L.-S. Young, 'Periodic points and topological entropy of one dimensional maps', in Global theory of dynamical systems 810: Lecture Notes in Math (Springer-Verlag, New York, 1980).

[3] U. Burkart, 'Interval mapping graphs and periodic points of continuous functions', J. Combin Theory Ser B 32 (1982), 57-68.

[4] B. Byers, 'Periodic points and chaos for expanding maps of the interval', Bull. Austral. Math. Soc. 24 (1981), 79-83.

[6] B.-S. Du, 'The minimal number of periodic orbits of periods guaranteed in Sharkovskii's theorem', Bull. Austral. Math. Soc. 31 (1985), 89-103.

[6] B.-S. Du, 'A note on periodic points of expanding inaps of the interval', Bull. Austral. Math. Soc. 33 (1986), 435-447.

[7] B.-S. Du, 'An example of a bifurcation from fixed points to period 3 points', Nonlinear Anal 10 (1986), 639-641.

[8] B.-S. Du, 'Examples of expanding maps with some special properties', Bull. Austral. Math. Soc. 36 (1987), $469-474$.

[9] C.-W. Ho and C. Morris, 'A graph theoretic proof of Sharkovsky's theorem on the periodic points of continuous functions', Pacific J. Math. 86 (1981), 361-370. 
[10] S. Ito, S. Tanaka and H. Nakada, 'On unimodal linear transformations and chaos $I$ ', Tokyo $J$. Math. 2 (1979), 221-239.

[11] T.-Y. Li, M. Misiurewicz, G. Pianigiani and J.A. Yorke, 'Odd chaos', Phys. Lett. A 87 (1982), 271-273.

[12] T.-Y. Li, M. Misiurewicz, G. Pianigiani and J.A. Yorke, 'No division implies chaos', Trans. Amer. Math. Soc. 273 (1982), 191-199.

[13] M. Osikawa and Y. Oono, 'Chaos in $C^{0}$-endomorphism of interval', Publ. Res. Inst. Math. Sci. Kyoto Univ. 17 (1981), 165-177.

[14] A.N. Sharkovskii, 'Coexistence of cycles of a continuous map of the line into itself, Ukain. Mat. Zh 61 (1964), 61-71. (Russian).

[15] P. Stefan, 'A theorem of Sharkovsky on the existence of periodic orbits of continuous endomorphisms of the real line', Comm. Math. Phys. 54 (1977), 237-248.

[10] P.D. Straffin Jr, 'Periodic points of continuous functions', Math. Mag. 51 (1978), 99-105.

[17] Z.-H. Zhang, 'Periodic points and chaos for expanding self-maps of the interval', Bull. Austral. Math. Soc. 31 (1985), 439-443.

Institute of Mathematics

Academia Sinica

Nankang

Taipei, Taiwen, 11529

Republic of China 